\title{
INNER NO OUTER regulates abaxial- adaxial patterning in Arabidopsis ovules
}

\author{
Jacinto M. Villanueva, ${ }^{1,3}$ Jean Broadhvest, ${ }^{1}$ Bernard A. Hauser, ${ }^{1,4}$ Robert J. Meister, ${ }^{1}$ Kay Schneitz, ${ }^{2}$ \\ and Charles S. Gasser ${ }^{1,5}$
}

${ }^{1}$ Section of Molecular and Cellular Biology, University of California, Davis, California 95616 USA; ${ }^{2}$ Institut für Pflanzenbiologie, Universitaet Zürich, CH-8008 Zürich, Switzerland

\begin{abstract}
The Arabidopsis INNER NO OUTER (INO) gene is essential for formation and asymmetric growth of the ovule outer integument. INO encodes a member of the newly described YABBY family of putative transcription factors that contain apparent $\mathrm{Cys}_{2}-\mathrm{Cys}_{2}$ zinc-finger domains and regions of similarity to the high mobility group (HMG) transcription factors. In wild-type plants, INO is expressed specifically on one side of the central region of each ovule primordium in the cells that give rise to the outer integument. Alterations in the INO expression pattern in mutant backgrounds implicate INO as a positive regulator of its own expression, and $A N T, H L L, B E L 1$, and $S U P$ as direct or indirect negative regulators that help to establish the spatial pattern of INO expression. We hypothesize that INO is necessary for polarity determination in the central part of the ovule. Maintenance of polarity in other parts of ino ovules indicates the existence of additional regulators and provides further evidence that the ovule is a compound structure.
\end{abstract}

[Key Words: Polarity; transcription factor; YABBY; INO; plant development; integument]

Accepted September 24, 1999; revised version accepted October 19, 1999.

Consisting of only four morphological parts, the Arabidopsis ovule is a relatively simple structure that lends itself to the study of genetic regulation of pattern formation and organogenesis in plants (for review, see Gasser and Robinson-Beers 1993; Reiser and Fischer 1993; Angenent and Colombo 1996; Gasser et al. 1998; Schneitz et al. 1998b). Although initiating as a radially symmetrical primordium, the developing ovule subsequently exhibits differences between the side toward the apex of the carpel (adaxial) and the side toward the base of the carpel (abaxial)—resulting in a bilaterally symmetrical structure. Asymmetric development is most apparent in the outer integument (one of two sheathing structures) and in the funiculus (supporting stalk). The outer integument grows extensively only on the abaxial side of the ovule, and the funiculus curves in the abaxial direction. Two genetic loci involved in asymmetric development of the outer integument have been described. In addition to effects on flower development (Schultz et al. 1991; Bowman et al. 1992), the SUPERMAN (SUP) gene is essential for suppressing adaxial growth of the outer integument (Gaiser et al. 1995), and mutations in this gene lead to nearly equal outer integument growth on both sides of the ovule. In contrast, inner no outer (ino) mutations can lead to an absence of outer integument

Present addresses: ${ }^{3}$ Department of Molecular Biology, Massachusetts General Hospital, Boston, Massachusetts 02114 USA; ${ }^{4}$ Department of Botany, University of Florida, Gainesville, Florida 32611 USA.

${ }^{5}$ Corresponding author.

E-MAIL csgasser@ucdavis.edu; FAX (530) 752-3085. growth on both sides of the ovule primordium, implicating INO as a positive regulator of integument growth or a determinant of polarity (Gaiser et al. 1995; Baker et al. 1997; Schneitz et al. 1997).

In angiosperms, bilateral symmetry is also a common characteristic of leaves, floral organs, and often whole flowers. In these structures, bilateral symmetry results from significant differences in development between the adaxial (toward the shoot apex) and abaxial (away from the shoot apex) sides. Recently, several genes involved in establishing these abaxial-adaxial patterns have been identified. The cycloidea (cyc) and dichotoma (dich) genes of Antirrhinum majus are expressed on the adaxial sides of flowers, where they specify adaxial floral development (Luo et al. 1996). In contrast, phantastica (phan), which appears to be essential for identity of the adaxial leaf surface in this same species, is expressed throughout the leaf (Waites et al. 1998), and must, therefore, require additional asymmetrically distributed factors for its activity.

In Arabidopsis thaliana, vegetative structures of the phabulosa-1d mutant are radially symmetrical, apparently as a result of adaxialization, and PHABULOSA may be one determinant of abaxial cell fate in this species (McConnell and Barton 1998). A recently described family of Arabidopsis genes, the YABBY genes, encoding putative transcription factors (Bowman and Smyth 1999; Kumaran et al. 1999; Sawa et al. 1999; Siegfried et al. 1999|, participate in determination of abaxial identity in a variety of organs. Three members of this family, FILAMENTOUS FLOWER (FIL), YABBY2 (YAB2), and YAB3, 
are expressed exclusively in the abaxial sides of cotyledons, leaves, and floral organs (Sawa et al. 1999; Siegfried et al. 1999). FIL and YAB3 were shown to be determinants of abaxial identity in these organs (Sawa et al. 1999; Siegfried et al. 1999). CRABS CLAW (CRC), another member of this family, has roles in nectary development and carpel architecture (Bowman and Smyth 1999|. CRC is expressed in nectaries and on the abaxial (external) face of carpels. Its role in abaxial development became apparent in the presence of second-site-enhancer mutations that cause carpels of crc mutants to exhibit partial adaxialization (Eshed et al. 1999).

Herein we describe the isolation and characterization of the Arabidopsis INO gene. We show that INO is a member of the YABBY gene family, and that, like the other family members, its asymmetric pattern of expression is closely associated with asymmetric development. Changes in the accumulation patterns of INO mRNA in different ovule mutant backgrounds identify several putative regulators of INO expression. The new data enable the formulation of models for the roles of both INO and SUP in regulation of polar development in ovules.

\section{Results}

\section{Phenotypic effects of ino mutations}

In wild-type plants, ovule primordia arise from the placental regions on the interior surfaces of the carpels as radially symmetrical finger-like projections. As the primordia grow, the funiculi bend toward the septum and the base of the carpel (the abaxial direction; Fig. 1A,B). The inner integument is initiated from a symmetrical ring of cells encircling the central part of the ovule primordium, whereas the outer integument initiates from cells on the abaxial side of the ovule primordium (Fig. 1A). The zone of integument initiation (the chalaza) separates the distal region (nucellus) from the proximal region (funiculus) of the developing ovule. The inner integument continues to grow symmetrically (Fig. 1B). The outer integument exhibits marked asymmetric growth throughout its development, growing extensively on the abaxial side, and only to a very limited extent on the adaxial side (Fig. 1B). The resulting hood-shaped structure covers both the inner integument and the nucellus (Fig. 1C).

As described previously (Baker et al. 1997; Schneitz et al. 1997), in strong ino alleles (i.e., ino-1 and ino-2) the outer integument fails to initiate on the abaxial side of the ovule, and subsequent outer integument development is not observed (Fig. 1D-F). However, a protuberance appears on the adaxial side of the ovule and undergoes limited expansion (Fig. 1D-F). In a newly identified weaker allele, ino-4, the outer integument initiates in the correct location on the abaxial side of an ovule primordium (Fig. 1G). However, subsequent growth of the outer integument is reduced as compared with wild type, and the outer integument fails to completely enclose the inner integument and nucellus (Fig. 1H-J). The adaxial protuberance observed in ino-1 and ino-2 was also observed in ino- 4 but was partially covered by the reduced outer integument (Fig. 1I,J). ino mutations caused no vis-

Figure 1. Scanning electron micrographs of wild-type and ino ovules. In all panels, the base of the ovary (and therefore, the abaxial side of the ovules) is at left. Bars, 10 um. (A) Wild-type ovules at stage 2-III (stages according to Schneitz et al. 1995). The nucellus, funiculus, and inner and outer integument primordia are visible. The outer integument initiates on the abaxial side of the wild-type ovule. (B) Wildtype ovules at stage 3-I. The outer integument encloses most of the inner integument and nucellus. $(C)$ Wild-type ovules at anthesis (stage 4-I). The outer integument completely covers the inner integument and nucellus. $(D)$ ino-1 ovules at stage 2-III. The outer integument on the abaxial side of the ovule is absent. A protuberance (arrowheads) forms on the adaxial side of each ino-1 ovule. (E) ino-1 ovules at stage 3-I. $(F)$ ino- 1 ovules at stage 4-I. The inner integument completely covers the nucellus and the adaxial protuberance (arrowheads) is enlarged. $(G)$ ino-4 ovules at stage 2-III. Initiation of the outer integuments on the abaxial sides is apparent (arrows). (H) ino-4
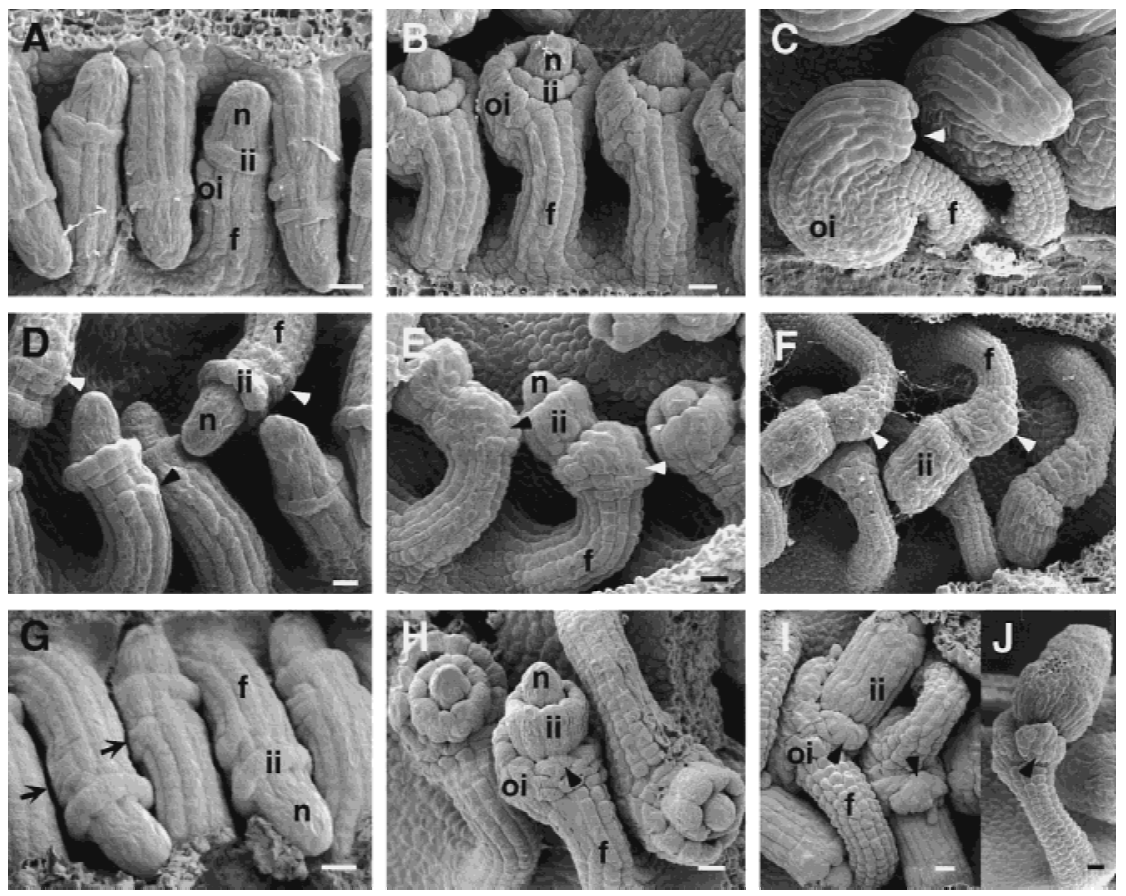

ovules at stage 3-I. The outer integuments are reduced relative to wild type. The adaxial protuberance is visible on some ovules (arrowhead). (I,J) ino-4 ovules at stage 4-I. The outer integuments are reduced relative to wild type. The adaxial protuberance (arrowheads) protrudes between the inner and outer integuments. (f) funiculus; (ii) inner integument (primordium); (n) nucellus; (oi) outer integument (primordium). 
ible alterations in other aspects of ovule, flower, or vegetative development (Fig. 1; Baker et al. 1997; Schneitz et al. 1997).

\section{Positional cloning of INO}

Because all available ino alleles derived from chemically mutagenized populations, we used a positional-cloning approach to isolate the INO gene. The physical position of INO was refined to within a region containing four putative genes (numbered 5-8) on two overlapping cosmid clones (cJMV41 and cJMV43). The sequences of genes 7 and 8 amplified from the genomes of mutant lines revealed putative mutations only in gene 7 for all three alleles (Fig. 2A,B). The independently isolated ino-1 and ino-2 mutants had a G-to-A transition near the splice acceptor site of intron 5 (of six total introns), and a similar transition was found near the intron 4 splice acceptor site in ino-4 (Fig. 2A,B). Gene 7 RT-PCR products were generated and sequenced from RNA of both wild-type strains and the three ino alleles. When compared with wild type, cDNAs from ino-1 and ino-2 included 11 additional nucleotides due to utilization of an aberrant splice-acceptor site created by the mutation (Figure 3B). This resulted in a frameshift in translation of the carboxy-terminal region of the predicted protein, replacing the last 59 amino acids with 75 essentially random residues. The mutation in the weak ino- 4 allele formed a new splice-acceptor site for intron 4, causing the inclusion of 15 additional nucleotides in the mRNA (Fig. 2B). These nucleotides translate into a five-aminoacid insertion, leaving the rest of the protein in frame. In all cases, the unambiguous sequence obtained directly from the RT-PCR products indicated that essentially all of the mRNA in the mutants derived from use of the

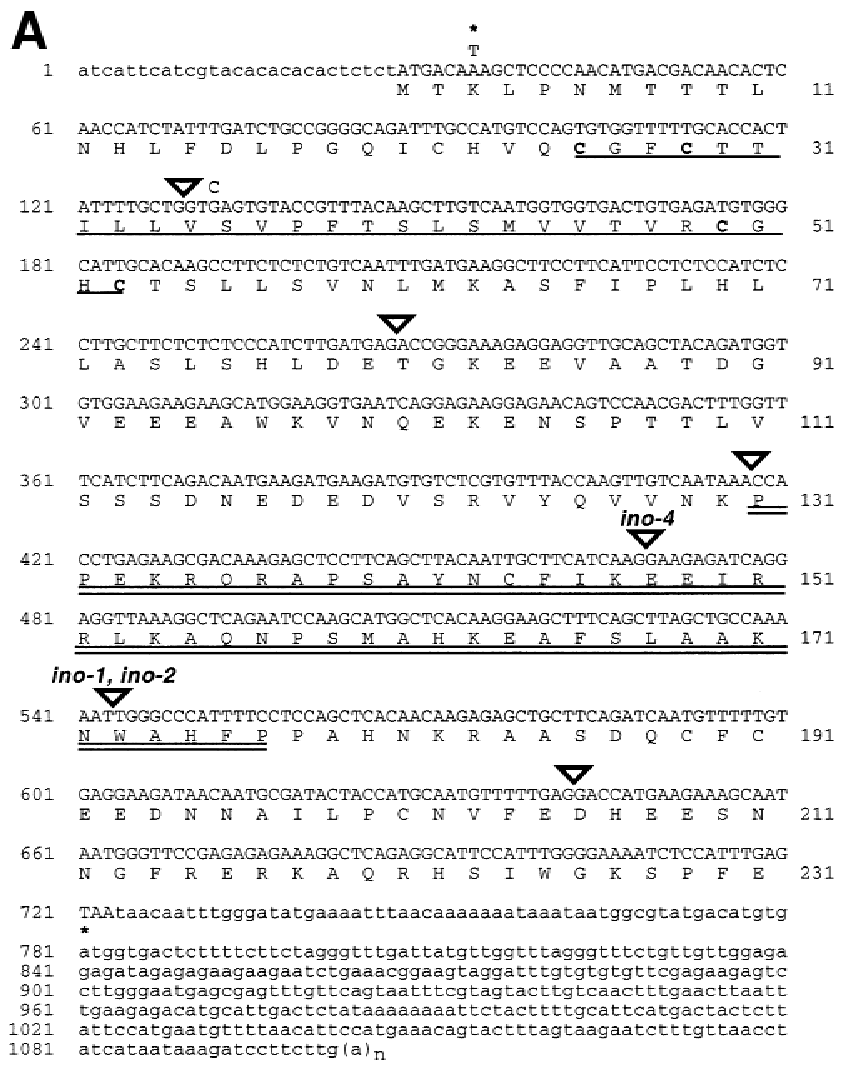

B

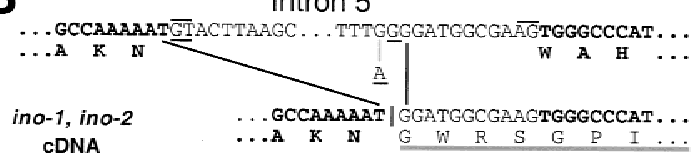

Intron 4

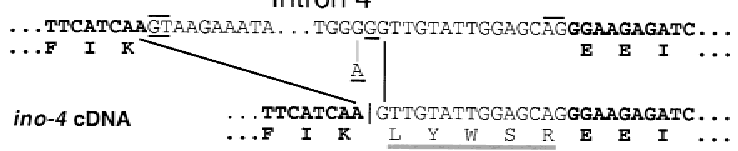

C

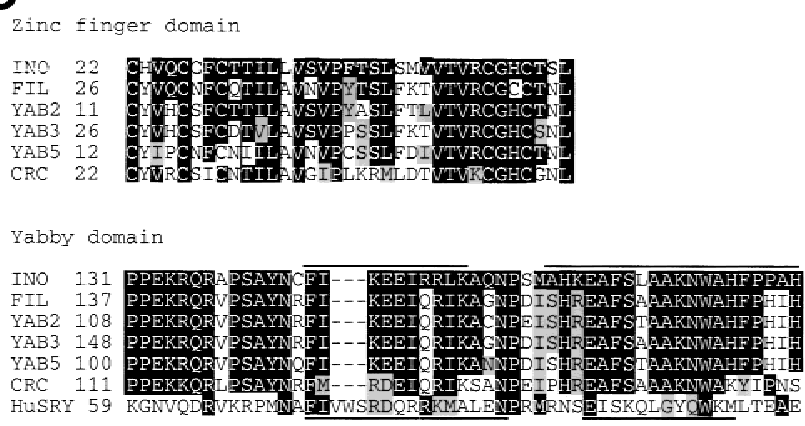

Figure 2. INO DNA and protein sequences. (A) INO cDNA and predicted protein sequence (Co-3 ecotype). Coding region of cDNA is shown in uppercase and $5^{\prime}$ - and $3^{\prime}$-untranslated regions are in lowercase. Nucleotide differences in the coding region of the Ler ecotype are shown above the cDNA sequence. Single and double underlines indicate the putative zinc finger and YABBY domains, respectively. Conserved cysteine residues in the putative zinc finger region are in boldface type. $(\nabla)$ Intron locations, and introns containing the ino-1, ino-2, and ino-4 mutations are indicated. (Asterisks) Stop codons. (B) Mutations lead to altered splicing. Wild-type genomic sequences of introns 5 and 4, with some flanking exon sequence, are illustrated at top of each panel. Wild-type exons and the amino acids they encode are shown in boldface and donor (GT) and acceptor (AG) intron splice sites are overlined. Vertical gray bars indicate G-to-A transitions identified in the mutant genomic sequences. Mutant cDNA sequences are illustrated below each genomic sequence. Mutant donor and acceptor sites are indicated by black underlines. Alterations in the protein sequence resulting from aberrant splicing in the mutants are indicated by gray underlines. $(C)$ Alignment of conserved putative zinc finger (top) and YABBY (bottom) domains. Black boxes indicate identical and gray boxes indicate conserved residues between INO and other proteins. Broken lines indicate gaps in the sequences. The overline indicates predicted $\alpha$-helical domains in INO (by the method of Chou et al. 1978); the underline indicates $\alpha$ helices in SRY (Werner et al. 1995). 


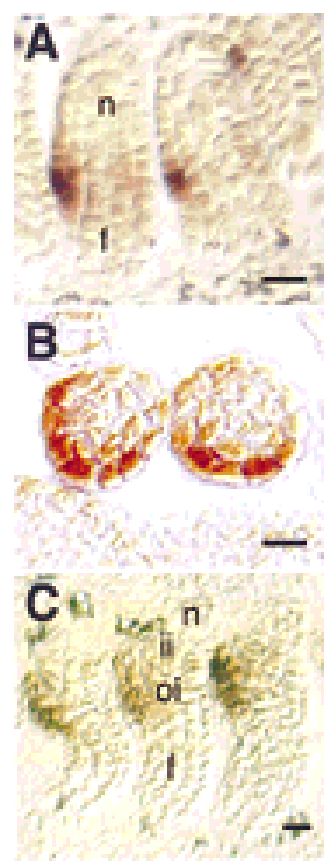

Figure 3. Histochemical detection of in situ hybridization with an INO antisense RNA probe in wild-type ovules. In all panels, the base of the carpel and the abaxial side of the ovules, is at left. Bars, $10 \mu \mathrm{m} .(A, B)$ Stage 2-I ovule primordia (before emergence of integuments). (A) Longitudinal sections show INO mRNA at the site of origination of the outer integument on the abaxial sides of three ovule primordia (two pointing upward and one pointing downward). (B) Cross sections show that INO mRNA is confined to a semicircular collar of cells corresponding to the site of initiation of the outer integument. $(C)$ Longitudinal section of ovules at stage 2-II. INO mRNA is in the abaxial layers of only the outer integument primordium. (f) Funiculus; (ii) inner integument primordium; (n) nucellus; (oi) outer integument primordium.

mutant splice junctions (data not shown). These data were consistent with gene 7 being INO.

\section{Complementation of ino-1 by transformation}

Cosmid cJMV41, which contains gene 7, was used for in planta transformation. Homozygous ino-1 plants containing cJMV41 displayed the Ino ${ }^{+}$phenotype, demonstrating that this cosmid contained INO. A second construct made of the gene $7 \mathrm{cDNA}$ flanked by $\sim 2.3 \mathrm{~kb}$ of $5^{\prime}$ and $2.0 \mathrm{~kb}$ of $3^{\prime}$ gene 7 genomic DNA was transferred into plants segregating for ino-1. Five of six independent transformants homozygous for ino-1 were Ino ${ }^{+}$, showing that the gene 7 cDNA transgene complements ino-1. Together, these data demonstrate that gene 7 is INO.

INO encodes a protein with zinc-finger and HMG-like domains

The Co-3 INO cDNA encodes a 231-amino-acid protein that includes a putative $\mathrm{Cys}_{2}-\mathrm{Cys}_{2}$ zinc-finger domain near the amino terminus, and a high mobility group (HMG)-like domain (Landsman and Bustin 1993; Grosschedl et al. 1994; Baxevanis and Landsman 1995) near the carboxyl terminus (Fig. 2A). The presence of these two domains is characteristic of the recently described YABBY family of putative transcription factors (Bowman and Smyth 1999; Sawa et al. 1999; Siegfried et al. 1999). The HMG-like domain of these proteins, designated the YABBY domain (Bowman and Smyth 1999), has sequence similarity to DNA-binding domains of HMG transcription factors such as the human SRY protein (Fig. 2C; Werner et al. 1995). The HMG-like domain of SRY consists of three $\alpha$-helices separated by turns, with the first two helices binding directly to DNA (Werner et al. 1995). The YABBY domains align with the first two helices of this motif and are predicted to form two $\alpha$-helices separated by a turn (Fig. 2C; data not shown).

INO sequences outside of the two conserved domains show no special similarity in amino acid composition or length to the corresponding regions of other Arabidopsis YABBY proteins (Siegfried et al. 1999; data not shown). The region of INO between the putative zinc finger and YABBY domains is hydrophilic and acidic $(21 \%$ glutamate plus aspartate), whereas in other YABBY proteins this region can be, for example, proline rich (YAB3; Siegfried et al. 1999), or serine rich (CRC; Bowman and Smyth 1999).

We note that a sequence difference in the Landsberg erecta (Ler) ecotype introduces a stop codon at the fourth codon of the conceptual translation (Fig. 2A). This indicates that in this ecotype (and possibly in Co-3 as well) translation must initiate at the second start codon, producing a protein that is six amino acids shorter at the amino terminus.

\section{Expression pattern of INO in wild-type ovules}

The spatial distribution of INO transcript accumulation was examined by in situ hybridization with an INO antisense probe (omitting the YABBY domain). INO mRNA was first detected in a group of $\sim 15$ epidermal cells on the abaxial half of each ovule primordium, prior to visible emergence of the integuments [stage 2-I of ovule development (Schneitz et al. 1995; Fig. 3A,B)]. These expressing cells correspond to the site of outer integument initiation (Fig. 3A,B). At stage 2-II/III, when outer and inner integument primordia are visible, INO mRNA was detected only in the outer integument on the abaxial side of the ovule primordium (Fig. 3C). At anthesis, INO mRNA was no longer present at detectable levels (data not shown). INO mRNA was not apparent in any other floral structures, although some putative signal was seen in all cells of globular, but not later stage embryos (data not shown). No hybridization was detected with the INO sense probe (data not shown).

\section{INO expression in mutant backgrounds}

We were unable to detect INO mRNA in ovules of the strong ino-1 allele by in situ hybridization, indicating 
that levels of accumulation in this mutant must be substantially lower than in wild type. INO mRNA was detected in the weaker ino-4 allele in the same pattern observed in wild-type ovules (data not shown). The relatively weak signal in these hybridizations indicated that INO mRNA may be accumulating to lower levels in ino-4 than in wild type.

In huellenlos-1 (hll-1) mutant ovules, only rudimentary integumentary ridges emerge from the chalaza (Fig. 4A; Schneitz et al. 1998a). Development ceases shortly thereafter, and cells of the nucellus commonly degenerate. INO transcripts were observed both in the chalaza and in the nucellus of $h l 1-1$ ovules (Fig. 4B). INO mRNA

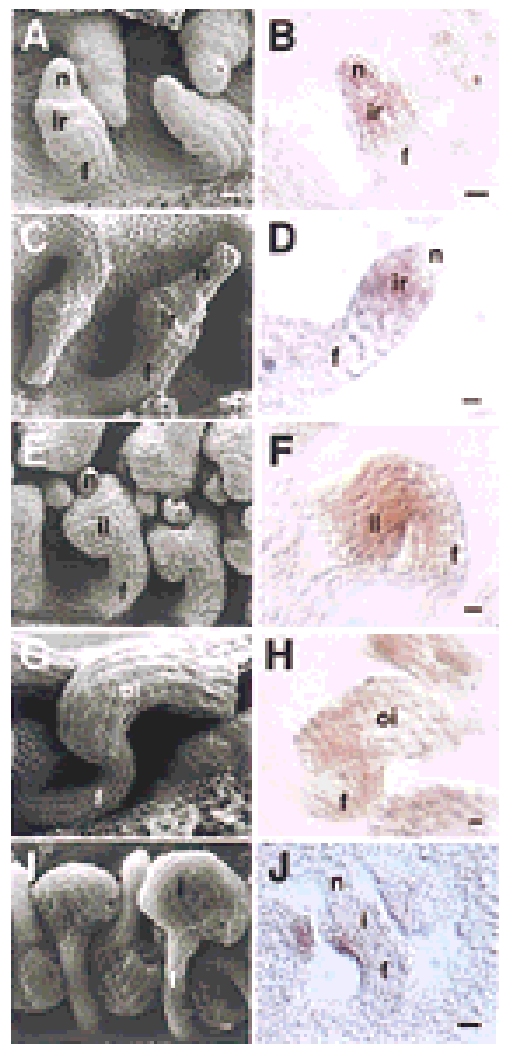

Figure 4. Distribution of $I N O$ mRNA in mutant ovules. Scanning electron micrographs $(A, C, E, G, I)$ and in situ hybridizations with an INO antisense RNA probe $(B, D, F, H, J)$ of mutant ovules. In all panels, the base of the carpel and the adaxial side of the ovules is at left. Bars, $10 \mu \mathrm{m} .(A, B)$ Stage 4-I hll-1 ovules. INO mRNA is detected in the integumentary ridge and nucellus but not in the funiculus. Note that the ridge is not evident in all ovules. $(C, D)$ Stage 4-I ant-4 ovules. INO mRNA is detected throughout the integumentary ridge. $(E, F)$ Stage 4-1 bel1-1 ovules. INO mRNA is detected uniformly throughout the integument-like structure of bel1 ovules. $(G, H)$ Stage 4-I sup-5 ovules. INO mRNA is detected on both sides of the base of the outer integument and in the funiculus. $(I)$ Stage 4-I and $(J)$ stage 2-III ats ovules. ats ovules have only a single integument (LeonKloosterziel et al. 1994). During early development of this structure, ino mRNA was detected only in the basal half of the abaxial side of the single integument primordium. (ir) Integumentary ridge; (n) nucellus; (f) funiculus; (il) integument-like structure; (i) single integument; (oi) outer integument. was not specific to the abaxial side of the ovule, but was evenly distributed across the chalaza and nucellus.

In the strong aintegumenta-4 (ant-4) mutant, in which the integuments are almost completely absent (Fig. 4C; Elliott et al. 1996; Klucher et al. 1996; Baker et al. 1997; Schneitz et al. 1998a), INO mRNA was detected only in the chalaza, in which it was evenly distributed along the abaxial-adaxial axis (Fig. 4D).

In bell1-1 (bel1-1) ovules, a single integument-like structure emerges from the chalaza (Fig. 4E; RobinsonBeers et al. 1992). INO transcript was uniformly distributed throughout the integument-like structure, and also extended into the funiculus of bel1-1 ovules (Fig. 4F).

In sup mutants, following apparently normal formation of an asymmetric outer integument primordium, the outer integument grows nearly equally on all sides of the ovule, resulting in a long tubular structure (Fig. 4G; Gaiser et al. 1995). In sup-5 ovules, the zone of INO transcript accumulation extends across both sides of the outer integument and down into the funiculus (Fig. 4H).

In aberrant testa shape (ats) mutants, a single integumentary structure is observed in place of the two integuments (Fig. 4I; Leon-Kloosterziel et al. 1994). This single integument has properties of both inner and outer integuments, and has been hypothesized to result from fusion between these two structures (Leon-Kloosterziel et al. 1994). In ats ovules, the INO transcript is detected only in the proximal half of the single integumentary structure, but its distribution along the abaxial-adaxial axis is as in wild type (Fig. 4J).

\section{Discussion}

INO is a member of the YABBY gene family

The YABBY genes encode a newly defined class of putative plant transcription factors (Bowman and Smyth 1999; Kumaran et al. 1999; Sawa et al. 1999; Siegfried et al. 1999). The conserved $\mathrm{Cys}_{2}-\mathrm{Cys}_{2}$ putative zinc-binding and HMG-like domains of INO make it a member of the YABBY gene family. The patterns of mRNA accumulation of four other YABBY genes have been described (Bowman and Smyth 1999; Sawa et al. 1999; Siegfried et al. 1999), and INO is the only member that shows expression in ovules. INO is present in the recently released genomic survey sequence of BAC clone F26F24 and this sequence was referred to previously as YABBY 4 in Siegfried et al. (1999).

The demonstrated zinc-binding activity of FIL (Sawa et al. 1999/ supports the hypothesis that the conserved $\mathrm{Cys}_{2}-\mathrm{Cys}_{2}$ domains of YABBY proteins represent zincbinding structures. There is significant conservation of amino acid residues beside the cysteines among these domains in the different YABBY proteins (Fig. 2B; Bowman and Smyth 1999; Sawa et al. 1999; Siegfried et al. 1999|. However, the absence of significant sequence similarity to previously described zinc finger domains indicates that the YABBY proteins may define a structurally distinct class of zinc-binding proteins. Regions outside of the zinc finger and YABBY domains exhibit 
little or no sequence conservation among most members of the YABBY family, and may define functional specificity.

\section{ino mutant effects}

On the basis of the phenotypes of strong ino alleles, we had hypothesized previously that the protuberance forming on the adaxial side of ino ovules represented an abortive outer integument in an incorrect location (Baker et al. 1997). The presence of both an outer integument and the adaxial protuberance in the newly characterized ino-4 mutant leads us to suggest other hypotheses. One alternative hypothesis is that the adaxial protuberance results from abnormal manifestation (due to the absence of an outer integument) of a normal process of ovule development. In wild-type Arabidopsis ovule development, following initiation of both integuments, there is subepidermal cell division on the adaxial side of the ovule in the region corresponding to the point of attachment of the two integuments (see, e.g., Fig. 2A of Robinson-Beers et al. 1992). We hypothesize that in the absence of outer integument development, this same cell division could initiate earlier and form the exposed abaxial protuberance in ino-1. Thus, it is possible that the only direct effect of a strong ino mutation is elimination of the outer integument. This is consistent with our observation that INO mRNA accumulates only in this structure. Although other possibilities remain, the phenotypic effects of severe ino mutations can be explained as primary and secondary results of absence of outer integuments.

\section{The role of INO in outer integument development}

The specific accumulation of INO mRNA in the cells giving rise to the outer integument suggest a simple model for the action of INO in which it acts as a general promoter of growth in the region in which it is expressed. The absence of INO activity in strong ino mutants would eliminate this influence, leading to a failure of the outer integuments to expand. This model is also consistent with our observation that in sup mutants, the near uniform expression of INO across the outer integument correlates with uniform growth of this structure on all sides of the Sup ${ }^{-}$ovule. Thus, the effects of sup mutations on ovule development may result only from changes in INO expression, consistent with ino being epistatic to sup in ovule development (Gaiser et al. 1995). INO cannot be sufficient to promote growth but must require other factors such as ANT, because in ant mutants there is no growth from the chalazal region despite significant INO expression (Fig. 4D). The need for other factors, which could be integument specific, could explain the lack of aberrant growth from the nucellus and funiculus in hll, bell, and sup mutants in which INO is expressed in these structures (Fig. 4).

The roles of other YABBY genes in plant development suggest an alternative model for the role of INO. Expres- sion of $C R C, Y A B 2, Y A B 3$, and FIL has been shown to be on the abaxial sides of leaves and primary floral organs, and $C R C, Y A B 3$, and FIL have been shown to be important promoters of abaxial identity in these structures (Bowman and Smyth 1999; Eshed et al. 1999; Sawa et al. 1999; Siegfried et al. 1999). The outer integument normally grows extensively on the abaxial side of the ovule, and shows almost no growth on the adaxial side (Fig. 1). If INO is necessary for abaxial identity in the part of the chalaza giving rise to the outer integument, then its absence would lead to adaxialization of this region. Because limited growth is characteristic of the adaxial side of the outer integument, this would result in limited, or no outer integument growth on either side of the ovule primordium. The model can also explain the sup mutant phenotype, in which expansion of INO expression to the adaxial side of the ovule would lead to abaxialization of this surface and more symmetrical growth of the integument. Because this second model hypothesizes INO to be only a determinant of polarity in a developing integument, the model is consistent with the absence of phenotypic effects of ectopic INO expression observed in the nucellus and funiculus in bel1, hll, and sup ovules.

The apparent conservation of function of YABBY genes in polar identity determination leads us to favor the second model in which INO is a primary determinant of abaxial identity of a part of the chalaza including the outer integument. We note that our definition of abaxial side of an ovule is only by analogy to the abaxial side of leaves (Gaiser et al. 1995). Thus, our model hypothesizes only a conservation of YABBY function in polar identity, rather than in a specific side of a polar structure.

\section{Regulation of INO expression}

Changes in patterns of INO mRNA accumulation in mutant backgrounds indicate roles for SUP, HLL, ANT, and $B E L 1$ in processes regulating INO expression. SUP has been shown previously to have a cadastral function in specification of the inner boundary of expression of the APETALA 3 (AP3) floral organ identity gene (Schultz et al. 1991; Bowman et al. 1992; Sakai et al. 1995). There are a number of striking parallels between the role of SUP in regulation of $A P 3$ and $I N O$. For both genes, in sup mutants there is expansion of expression outside of the region of normal mRNA accumulation. Expression expands into the fourth floral whorl for AP3 (Bowman et al. 1992), and to the adaxial side of the ovule for INO (Fig. $4 \mathrm{H})$. For $A P 3$, the expanded zone of expression can explain most effects of sup mutations on floral organ identity (Jack et al. 1994; Sakai et al. 1995). However, changes in AP3 expression cannot account for sup effects on ovule development (Gaiser et al. 1995). As outlined above, we now show that the effects of sup mutations on ovule development may be completely mediated by the expansion of INO expression to the adaxial side of the ovule. We would therefore predict that ectopic expression of INO in ovules would lead to this same phenotype. 
In both floral organ identity and ovule development, the cells in which gene expression is repressed by SUP are adjacent to the cells in which SUP mRNA accumulates. SUP mRNA accumulates in the third whorl of floral organs, but inhibits expression of AP3 in the fourth whorl by an apparently cell nonautonomous mechanism (Sakai et al. 1995). In the ovule, SUP mRNA was observed only in a small region on the adaxial side of the funiculus just below the site of outer integument initiation (Sakai et al. 1995). However, it is in the adjacent chalazal region that SUP appears to repress INO expression. Therefore, it is possible that SUP negatively regulates INO in ovules and AP3 in flowers by a common cell nonautonomous mechanism.

The expansion of the zone of INO mRNA accumulation in hll, ant, and bel1 mutants (Fig. 4) implies that all three of these genes have roles in repressing INO expression in areas outside of the abaxial side of the chalaza. This is consistent with previous observations that all three mutations are epistatic to ino and apparently act earlier in ovule development (Baker et al. 1997; Gasser et al. 1998; Schneitz et al. 1998b). ANT and BEL1 are putative transcription factors (Reiser et al. 1995; Elliott et al. 1996; Klucher et al. 1996) and could directly regulate INO expression. However, neither protein could perform this function alone, because both are expressed throughout the chalaza (Reiser et al. 1995; Elliott et al. 1996; Klucher et al. 1996), including the region in which INO is normally expressed. They would have to act together with the products of other genes with more restricted or overlapping patterns of expression to suppress INO expression only on the adaxial side of the ovule primordium.

Expansion of the zone of INO expression could also be an indirect result of other effects of hll, ant, and bell mutations. These three genes have been proposed previously as regulators of proximal-distal patterning or growth (Reiser et al. 1995; Elliott et al. 1996; Klucher et al. 1996; Schneitz et al. 1998a), and disruption in any of these early acting regulators could interrupt establishment of the developmental zones within the ovule. Thus, our results may indicate a linkage between proximal-distal and abaxial-adaxial patterning in ovules.

Our inability to detect INO mRNA in ino-1 ovules by in situ hybridization indicates a dramatic reduction in mRNA levels in the mutant. Because the alteration in ino- 1 leads only to inclusion of 11 additional nucleotides in the INO transcript through a preferential splicing event, it is unlikely to have a significant negative effect on splicing efficiency or mRNA stability. However, interruption of the reading frame prior to the conserved tryptophan residue in the YABBY domain makes it likely that the mutant protein is inactive. The simplest explanation for the reduction of mRNA in the ino-1 mutant would be for INO to be a positive transcriptional regulator of INO. Because INO appears to be a transcription factor, this regulation could be direct. Thus, following initial activation of INO expression, the presence of active INO protein could maintain expression in the initially activated cells. Such autoregulation has been ob- served previously in maintenance of boundaries of expression for floral organ identity genes such as APETALA3 and PI (Goto and Meyerowitz 1994; Jack et al. 1994).

\section{Perspective}

The evidence presented above suggests that INO is involved in polarity of only one small region of the ovule primordium, the anlage to the outer integument. This contrasts with other characterized YABBY genes that appear to regulate abaxial-adaxial development in entire leaves or primary floral organs (Bowman and Smyth 1999; Sawa et al. 1999; Siegfried et al. 1999). This may indicate diversification of the YABBY family through expansion or contraction of ancestral expression patterns, but is more likely additional evidence that the ovule is a compound structure. The fossil record indicates that the structures of an angiosperm ovule have been added sequentially in a number of events that may be separated by hundreds of millions of years (Stewart 1983; Gasser et al. 1998). The precursor to INO may have become incorporated into ovule development at the origin of the outer integument, or with the possibly separate origin of asymmetry of this structure. Analysis of patterns of expression of INO orthologs in ancestral species could differentiate between these hypotheses. Integumentary fusion has been proposed as one mechanism for the derived origin of unitegmic ovules in the angiosperms (Bouman 1984). In ats mutants, the pattern of INO mRNA accumulation in only the proximal part of the single integument (Fig. 4J) supports the hypothesis that this mutation results in fused integuments. Examination of the expression of INO orthologs in unitegmic angiosperms could test the hypothetical origin of their ovule morphologies. Further analysis of INO thus promises not only to illuminate the regulation of ovule ontogeny but may also help us to understand the process of ovule evolution.

\section{Materials and methods}

\section{Plant material}

Isolation of ino-1 and ino-2 in the Ler background was described previously (Baker et al. 1997; Schneitz et al. 1997). ino-4 was isolated in the Columbia-3 (Co-3) background by similar methods, and was a kind gift of Imran Siddiqi (CCMB, Hyderabad, India). Mutants were backcrossed at least three times to wildtype plants of the parental ecotype prior to further analysis. Allelism was determined by genetic complementation tests (data not shown).

bel1-1 (Robinson-Beers et al. 1992), ant-4 (Baker et al. 1997), ats (Leon-Kloosterziel et al. 1994), hll-1 (Schneitz et al. 1998a), and sup-5 (Gaiser et al. 1995) were described previously in the cited references.

Plants were grown as described previously (Kranz and Kirchheim 1987; Robinson-Beers et al. 1992).

\section{Scanning electron microscopy}

Samples were prepared as described previously (Robinson-Beers et al. 1992) and were examined with a Hitachi (Tokyo, Japan) 
S3500N scanning electron microscope at an accelerating voltage of 5 or $10 \mathrm{kV}$. Images were acquired digitally and were edited for publication in Photoshop 4.0 (Adobe Systems, San Jose, CA).

\section{Genetic and physical mapping}

INO was mapped previously to chromosome 1 (Baker et al. 1997). More detailed mapping utilized DNA samples (Edwards et al. 1991) isolated from a total of $974 \mathrm{~F}_{2}$ plants from a cross of ino-1 (in the Ler background) to Co-3 wild-type plants. Informative recombinants were identified from this set by testing all samples for recombination between the cleaved amplified polymorphic sequence (CAPS) markers for the m235 (Hardtke and Berleth 1996) and CAULIFLOWER (CAL) (Kempin et al. 1995) loci, which flank INO and are separated by $\sim 2.3 \mathrm{cM}$. The $C A L$ CAPS marker was generated with gene-specific primers (a gift from Martin Yanofsky, University of California, San Diego) and digestion with SacI or DpnII. Genetic maps were determined with MapMaker 2.0 for Macintosh (Lander et al. 1987).

Further mapping utilized markers including g17286 (HindIII digest; Hauge et al. 1993), AthZFPG (Bell and Ecker 1994), and EST ve037 detecting two distinct but proximal loci (ve037E, EcoRV digestion, and ve037T, TaqI digestion), with these last two markers mapping 0.33 and $0.05 \mathrm{cM}$ north and south of ino, respectively.

Bacterial artificial chromosome (BAC) T29E11 was identified by probing the TAMU-BAC library (Choi et al. 1995) with a ${ }^{32}$ P-labeled ve037. T29E11 has a 70-kb insert and contains both ve037 polymorphisms. T29E11 was partially digested with Sau3A1 and DNA fragments from 17 to $25 \mathrm{~kb}$ were isolated by fractionation on a $10 \%-40 \%$ sucrose gradient and were subcloned into the BamHI site of the pOCA28 plant transformation cosmid vector (Olszewski et al. 1988). Cosmids were packaged in vitro (Stratagene, La Jolla, CA) and introduced into ves257 and JM101 bacterial strains. Comparison of restriction endonuclease digestion patterns and hybridization analysis with isolated ${ }^{32} \mathrm{P}$-labeled cosmid clones enabled the construction of a cosmid contig encompassing T29E11 (not shown). Additional RFLP markers were generated through hybridizations with the cosmids or cosmid subclones, allowing refinement of the position of INO to within two overlapping cosmids, cJMV41 and cJMV43.

For DNA sequence determination, cosmids cJMV41 and cJMV43 were partially digested with Sau3A1, fragments of $\sim 1-8$ $\mathrm{kb}$ were isolated by sucrose density gradient centrifugation (see above), and were subcloned into the BamH1 site of pUC119 (Vieira and Messing 1987). Cosmid end-rescue was performed by self-ligation following HindIII or EcoRI digestion, followed by PCR with primers to the COS site and the ColE1 replicon of the pOCA28 vector (Olszewski et al. 1988) (EcoRI primer, GCCGCTGTAAAGTGTTACGTTG; HindIII primer, GACGCAGGTATCGTATTAATTG). PCR products were purified and sequenced directly. Putative genes in this region, numbered 1-9, were identified with NetPlantGene (http://www.cbs.dtu.dk/ NetPlantGene.html, gene 5) or by alignment with cDNA sequences (other genes).

\section{Isolation of INO $c D N A s$}

An Arabidopsis (Ler) floral cDNA library (a gift of Detlef Weigel, Salk Institute, San Diego, CA) was screened with cJMV41 and cJMV43 insert DNA as probes. Two different cDNAs were isolated, one of which derived from INO (pJMV50). To determine the complete sequence of the $5^{\prime}$ region of the INO mRNA, 5' rapid amplification of cDNA ends (RACE; Frohman 1990) was performed according to Harper et al. (1990), except for the use of a gene specific primer complementary to bases 912-933 of the INO cDNA (Fig. 2A). PCR products were subcloned into pBluescript KS+ plasmid (Stratagene, La Jolla, CA) and transformed into JM101 cells.

To determine the sequence of INO mRNA in the mutant lines, first-strand cDNA was synthesized from total RNA isolated from wild-type Co-3 and the ino mutants. INO cDNAspecific primers corresponding to bases 32-51 and 912-933 in the INO cDNA (Fig. 2A) were used to amplify the INO-coding regions by $\mathrm{PCR}$, and the products were sequenced directly with these same primers.

\section{DNA sequence analysis}

DNA was sequenced with Big Dye terminators (Perkin-Elmer Corp., Norwalk, CT) and the Prism-377-XL system (PE Applied Biosystems, Foster City, CA). Sequences were assembled with Sequencher 3.0 software (GeneCodes Corp., Ann Arbor, MI) and Genetics Computer Group software (v. 9.1, Genetics Computer Group, Madison, WI). Database searches were performed at the National Center for Biotechnology Information web site (http:// www.ncbi.nlm.nih.gov/).

\section{Complementation by transformation}

Plasmid or cosmids were introduced into the ASE strain of Agrobacterium tumafaciens (Fraley et al. 1985) by triparental mating (Figurski and Helinski 1979), for in planta transformation of Arabidopsis (Bechtold and Pelletier 1998). cJMV41 was introduced into the Co-3 ecotype and transformants were selected on $50 \mu \mathrm{g} / \mathrm{ml}$ kanamycin and confirmed by gel blotting and hybridization with ${ }^{32} \mathrm{P}$-labeled pOCA2 8 vector. One transformant was crossed with pollen from a homozygous ino-1 (Ler) plant. Four of $16 \mathrm{~F}_{2}$ plants were homozygous for the Ler alleles of the m235 and AthZFPG markers, which are closely linked to and flank ino, indicating that they were homozygous for ino-1. Two of these plants were phenotypically Ino $^{+}$(consistent with segregation of the transforming DNA), demonstrating that the transgene could complement the ino-1 mutation.

The INO cDNA was inserted between the $5^{\prime}$ - $(2.3 \mathrm{~kb}$ extending to the first SalI site) and $3^{\prime}-(2 \mathrm{~kb}$ extending to the first EcoRI site) flanking genomic regions of INO. This genomic::cDNA chimera was inserted into the pMLBART (a gift from Kim Richardson, Hort Research, Auckland, NZ) transformation vector (Gleave 1992), resulting in plasmid pRJM33. pRJM33 was transformed into plants segregating for the ino-1 allele (in the Ler background) and the INO allele (in the Co-3 background). Transformants were selected for phosphinothricine (BASTA) resistance and verified by PCR reactions with gene-specific primers that amplify different sizes of fragments from the endogenous gene and the cDNA transgene. Transformants homozygous for the ino-1 allele (in the Ler background) were identified as those homozygous for the Ler allele of the m235 CAPS marker (0.33 cM north of ino).

\section{In situ hybridization}

Sections of plant tissue $8-10 \mu \mathrm{m}$ thick were prepared and hybridized as described previously (Vielle-Calzada et al. 1999) with the INO sense and antisense probes. Templates for INO probe preparation were constructed by subcloning a $\sim 450$-bp BamHI/ SacI fragment of pJMV50 including sequence encoding the zinc finger domain, but not the YABBY domain, into pBluescript KS+ plasmid vector digested with these same enzymes. The resulting plasmid, pJMV86, was linearized for synthesis of sense and antisense probes by digestion with $\mathrm{SacI}$ or XhoI, respectively. 
The 3'overhangs of SacI digestions were filled in with T4 DNA polymerase prior to probe synthesis. Digoxygenin-labeled probes were synthesized with the Genius RNA Labeling kit (Boehringer Mannheim Corp., Indianapolis, IN) according to the manufacturer's instructions. Sections were photographed on a Zeiss (Oberkochen, Germany) Axioplan microscope with differential interference contrast optics. Images were processed as described above.

\section{Acknowledgments}

We thank Linh Nguyen, Yin Shan Tai, Stephen Gross, Roderick Kumimoto, and Jordan Hay for technical assistance; Imran Siddiqi, Detlef Weigel, Martin Yanofsky, and Kim Richardson for generous gifts of materials; Karen Lee and George Coupland for sharing molecular marker data; Michael Dunlap and Rick Harris for assistance with SEM; Kay Robinson-Beers for SEM images; John Bowman and Ueli Grossniklaus for helpful advice on in situ hybridization; Sureshkumar Balasubramanian, Shawn Baker, Robert Kuzoff, Debra Skinner, Jessica McAbee, other past and present members of the Gasser laboratory, and members of the John Bowman laboratory for helpful discussions and suggestions. We thank the Arabidopsis Biological Resource Center at Ohio State for clones. This work was supported by the National Research Initiative Competitive Grants Program/U.S. Department of Agriculture (96-35304-3707), the National Science Foundation (NSF; IBN98-08395), grants from the Swiss National Science Foundation (31-42175.94, 31-53032.97), and the Kanton of Zürich to K.S., and an NSF Plant Cell Biology Training Grant fellowship to J.M.V.

The publication costs of this article were defrayed in part by payment of page charges. This article must therefore be hereby marked "advertisement" in accordance with 18 USC section 1734 solely to indicate this fact.

\section{Note}

Sequence data described in this paper have been submitted to GenBank under accession number AF195047.

\section{References}

Angenent, G.C. and L. Colombo. 1996. Molecular control of ovule development. Trends Plant Sci. 1: 228-232.

Baker, S.C., K. Robinson-Beers, J.M. Villanueva, J.C. Gaiser, and C.S. Gasser. 1997. Interactions among genes regulating ovule development in Arabidopsis thaliana. Genetics 145: 1109-1124.

Baxevanis, A.D. and D. Landsman. 1995. The HMG-1 box protein family: Classification and functional relationships. Nucleic Acids Res. 23: 1604-1613.

Bechtold, N. and G. Pelletier. 1998. In planta Agrobacteriummediated transformation of adult Arabidopsis thaliana plants by vacuum infiltration. Methods Mol. Biol. 82: 259266.

Bell, C.J. and J.R. Ecker. 1994. Assignment of 30 microsatellite loci to the linkage map of Arabidopsis. Genomics 19: 137144.

Bouman, F. 1984. The ovule. In Embryology of the angiosperms (ed. B.M. Johri), pp. 123-157. Springer-Verlag, New York, NY.

Bowman, J.L. and D.R. Smyth. 1999. CRABS CLAW, a gene that regulates carpel and nectary development in Arabidopsis, encodes a novel protein with zinc finger and helix-loop-helix domains. Development 126: 2387-2396.

Bowman, J.L., H. Sakai, T. Jack, D. Weigel, U. Mayer, and E.M.
Meyerowitz. 1992. SUPERMAN, a regulator of floral homeotic genes in Arabidopsis. Development 114: 599-615.

Choi, S., R.A. Creelman, J.E. Mullet, and R.A. Wing. 1995. Construction and characterization of a bacterial artificial chromosome library of Arabidopsis thaliana. Weeds World 2: 17-20.

Chou, P.Y. and G.D. Fasman. 1978. Prediction of the secondary structure of proteins from their amino acid sequence. Adv. Enzymol. 47: 45-148.

Edwards, K., C. Johnstone, and C. Thompson. 1991. A simple and rapid method for the preparation of plant genomic DNA for PCR analysis. Nucleic Acids Res. 19: 1349.

Elliott, R.C., A.S. Betzner, E. Huttner, M.P. Oakes, W.Q.J. Tucker, D. Gerentes, P. Perez, and D.R. Smyth. 1996. AINTEGUMENTA, an APETALA2-like gene of Arabidopsis with pleiotropic roles in ovule development and floral organ growth. Plant Cell 8: 155-168.

Eshed, Y., S.F. Baum, and J.L. Bowman. 1999. Distinct mechanisms promote polarity establishment in carpels of Arabidopsis. Cell 99: 199-209.

Figurski, D.H. and D.R. Helinski. 1979. Replication of an origincontaining derivative of plasmid RK2 dependent on a plasmid function provided in trans. Proc. Natl. Acad. Sci. 76: $1648-1652$.

Fraley, R.T., S.G. Rogers, R.B. Horsch, D.A. Eichholtz, J.S. Flick, C.L. Fink, N.L. Hoffmann, and P.R. Sanders. 1985. The SEV system: A new disarmed Ti plasmid vector for plant transformation. Bio/Technology 3: 629-635.

Frohman, M.A. 1990. RACE: Rapid amplification of cDNA ends. In PCR protocols, a guide to methods and applications. (ed. M. Innis, D. Gelfand, J. Sninsky, and T. White), pp. 28-38. Academic Press, San Diego, CA.

Gaiser, J.C., K. Robinson-Beers, and C.S. Gasser. 1995. The Arabidopsis SUPERMAN gene mediates asymmetric growth of the outer integument of ovules. Plant Cell 7: 333-345.

Gasser, C.S. and K. Robinson-Beers. 1993. Pistil Development. Plant Cell 5: 1231-1239.

Gasser, C.S., J. Broadhvest, and B.A. Hauser. 1998. Genetic analysis of ovule development. Annu. Rev. Plant Physiol. Plant Mol. Biol. 49: 1-24.

Gleave, A.P. 1992. A versatile binary vector system with a TDNA organisational structure conducive to efficient integration of cloned DNA into the plant genome. Plant Mol. Biol. 20: $1203-1207$.

Goto, K. and E.M. Meyerowitz. 1994. Function and regulation of the Arabidopsis floral homeotic gene PISTILLATA. Genes \& Dev. 8: 1548-1560.

Grosschedl, R., K. Giese, and J. Pagel. 1994. HMG domain proteins: Architectural elements in the assembly of nucleoprotein structures. Trends Genet. 10: 94-100.

Hardtke, C.S. and T. Berleth. 1996. Genetic and contig map of a 2200-kb region encompassing $5.5 \mathrm{cM}$ on chromosome 1 of Arabidopsis thaliana. Genome 39: 1086-1092.

Harper, J.F., L. Manney, N.D. DeWitt, M.H. Yoo, and M.R. Sussman. 1990. The Arabidopsis thaliana plasma membrane $\mathrm{H}(+)$-ATPase multigene family. Genomic sequence and expression of a third isoform. J. Biol. Chem. 265: 13601-13608.

Hauge, B.M., S.M. Hanley, S. Cartinhour, J.M. Cherry, H.M. Goodman, M. Koornneef, P. Stam, C. Chang, S. Kempin, L. Medrano, and E.M. Meyerowitz. 1993. An integrated genetic RFLP map of the Arabidopsis thaliana genome. Plant $J$. 3: 745-754.

Jack, T., G.L. Fox, and E.M. Meyerowitz. 1994. Arabidopsis homeotic gene APETALA3 ectopic expression - transcriptional and posttranscriptional regulation determine floral organ identity. Cell 76: 703-716. 
Kempin, S.A., B. Savidge, and M.F. Yanofsky. 1995. Molecular basis of the cauliflower phenotype in Arabidopsis. Science 267: 522-525.

Klucher, K.M., H. Chow, L. Reiser, and R.L. Fischer. 1996. The AINTEGUMENTA gene of Arabidopsis required for ovule and female gametophyte development is related to the floral homeotic gene APETALA2. Plant Cell 8: 137-153.

Kranz, A.R. and B. Kirchheim. 1987. Handling of Arabidopsis. In Arabidopsis information service, v. 24: Genetic resources in Arabidopsis (ed. A.R. Kranz), pp. 4.1.1-4.2.7. Arabidopsis Information Service, Frankfurt, Germany.

Kumaran, M.K., D. Ye, W.-C. Yang, M.E. Griffith, A.M. Chaudhury, and V. Sundaresan. 1999. Molecular cloning of ABNORMAL FLORAL ORGANS: A gene required for flower development in Arabidopsis. Sex. Plant Reprod. 12: 118-122.

Lander, E.R., P. Green, J. Abrahamson, A. Barlow, M. Daly, S.E. Lincoln, and L. Newburg. 1987. MAPMAKER: An interactive computer package for constructing primary genetic linkage maps of experimental and natural populations. Genomics 1: 174-181.

Landsman, D. and M. Bustin. 1993. A signature for the HMG-1 box DNA-binding proteins. BioEssays 15: 539-546.

Leon-Kloosterziel, K.M., C.J. Keijzer, and M. Koornneef. 1994. A seed shape mutant of Arabidopsis that is affected in integument development. Plant Cell 6: 385-392.

Luo, D., R. Carpenter, C. Vincent, L. Copsey, and E. Coen. 1996. Origin of floral asymmetry in Antirrhinum. Nature 383: 794-799.

McConnell, J.R. and K. Barton. 1998. Leaf polarity and meristem formation in Arabidopsis. Development 125: 2935-2942.

Olszewski, N.E., F.B. Martin, and F.M. Ausubel. 1988. Specialized binary vector for plant transformation: Expression of the Arabidopsis thaliana AHAS gene in Nicotiana tabacum. Nucleic Acids Res. 16: 10765-10782.

Reiser, L. and R.L. Fischer. 1993. The ovule and embryo sac. Plant Cell 5: 1291-1301.

Reiser, L., Z. Modrusan, L. Margossian, A. Samach, N. Ohad, G.W. Haughn, and R.L. Fischer. 1995. The BELL1 gene encodes a homeodomain protein involved in pattern formation in the Arabidopsis ovule primordium. Cell 83: 735-742.

Robinson-Beers, K., R.E. Pruitt, and C.S. Gasser. 1992. Ovule development in wild-type Arabidopsis and two female-sterile mutants. Plant Cell 4: 1237-1249.

Sakai, H., L.J. Medrano, and E.M. Meyerowitz. 1995. Role of SUPERMAN in maintaining Arabidopsis floral whorl boundaries. Nature 378: 199-203.

Sawa, S., K. Watanabe, K. Goto, E. Kanaya, E.H. Morita, and K. Okada. 1999. FILAMENTOUS FLOWER, a meristem and organ identity gene of Arabidopsis, encodes a protein with a zinc finger and HMG-related domains. Genes \& Dev. 13: $1079-1088$.

Schneitz, K., M. Hulskamp, and R.E. Pruitt. 1995. Wild-type ovule development in Arabidopsis thaliana: A light microscope study of cleared whole-mount tissue. Plant J. 7: 731749.

Schneitz, K., M. Hulskamp, S. Kopczak, and R. Pruitt. 1997. Dissection of sexual organ ontogenesis: A genetic analysis of ovule development in Arabidopsis thaliana. Development 124: $1367-1376$.

Schneitz, K., S.C. Baker, C.S. Gasser, and A. Redweik. 1998a. Pattern formation and growth during floral organogenesis: HUELLENLOS and AINTEGUMENTA are required for the formation of the proximal region of the ovule primordium in Arabidopsis thaliana. Development 125: 2555-2563.

Schneitz, K., S. Balasubramanian, and U. Schiefthaler. 1998b.
Organogenesis in plants: The molecular and genetic control of ovule development. Trends Plant Sci. 3: 468-472.

Schultz, E.A., F.B. Pickett, and G.W. Haughn. 1991. The FLO10 gene product regulates the expression domain of homeotic genes AP3 and PI in Arabidopsis flowers. Plant Cell 3: 12211237.

Siegfried, K.R., Y. Eshed, S.F. Baum, D. Otsuga, D.N. Drews, and J.L. Bowman. 1999. Members of the YABBY gene family specify abaxial cell fate in Arabidopsis. Development 128: $4117-4128$.

Stewart, W.N. 1983. Paleobotany and the evolution of plants. Cambridge University Press, New York, NY.

Vieira, J. and J. Messing. 1987. Production of single stranded plasmid DNA. Methods Enzymol. 153: 3-11.

Vielle-Calzada, J.-P., J. Thomas, C. Spillane, A. Coluccio, M.A. Hoeppner, and U. Grossniklaus. 1999. Maintenance of genomic imprinting at the Arabidopsis MEDEA locus requires zygotic DDM1 activity. Genes \& Dev. 13: 2971-2982.

Waites, R., H.R.N. Selvadurai, I.R. Oliver, and A. Hudson. 1998. The PHANTASTICA gene encodes a MYB transcription factor involved in growth and dorsoventrality of lateral organs in Antirrhinum. Cell 93: 779-789.

Werner, M.H., J.R. Huth, A.M. Gronenborn, and G.M. Clore. 1995. The molecular basis of human 46x,Y sex reversal revealed from the three-dimensional solution structure of the human Sry-DNA complex. Cell 81: 705-714. 


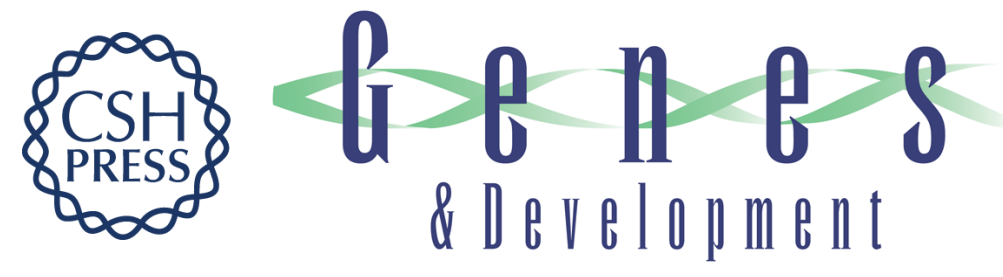

\section{INNER NO OUTER regulates abaxial - adaxial patterning in Arabidopsis ovules}

Jacinto M. Villanueva, Jean Broadhvest, Bernard A. Hauser, et al.

Genes Dev. 1999, 13:

\section{License}

Email Alerting Receive free email alerts when new articles cite this article - sign up in the box at the top Service right corner of the article or click here.

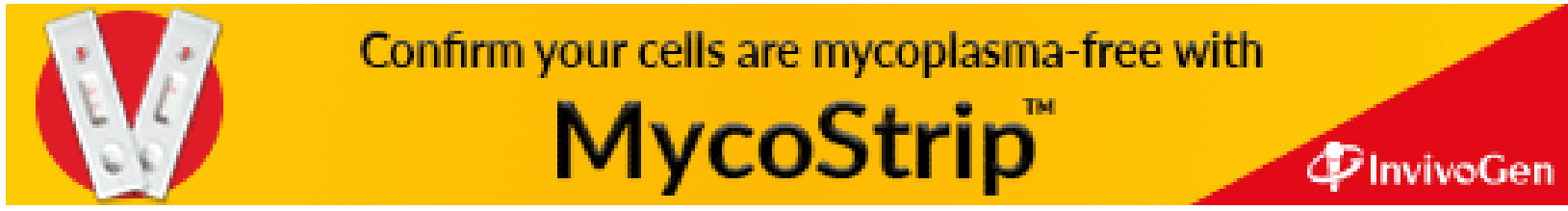

